\title{
Launching a Statewide COVID-19 Primary Care Hotline and Telemedicine Service
}

\author{
Anthony Cheng, MD, Heather Angier, PhD, MPH, Nathalie Huguet, PhD, \\ Deborah J. Cohen, PhD, Kellen Strickland, BSN, Emily Barclay, MS, \\ Eric Herman, MD, Craig McDougall, MD, Frances E. Biagioli, MD, Kam Pierce, MPA, \\ Carliana Straub, Bennett Straub, and Jennifer DeVoe, MD, DPhil
}

To respond to the COVID-19 pandemic and recover from its aftermath, primary care teams will face waves of overwhelming demand for information and the need to significantly transform care delivery.

Innovation: Oregon Health \& Science University's primary care team envisioned and implemented the CoVID-19 Connected Care Center, a statewide telephone "hotline" service.

Results: The hotline has taken more than $\mathbf{5 8 2 5}$ calls from patients in 33 of Oregon's 36 counties in less than 3 months. In preliminary survey data, $86 \%$ of patients said their questions were answered during the call, $90 \%$ would recommend this service, and $70 \%$ reported a reduction in stress levels about coronavirus. In qualitative interviews, patients reported their questions answered, short wait times, nurses spent time as needed, and appropriate follow-up was arranged.

Conclusion: Academic health centers may have the capacity to leverage their extensive resources to rapidly launch a multiphased pandemic response that meets peoples' need for information and access to primary care, while minimizing risk of infection and emergency department use and rapidly supporting primary care teams to make the necessary operational changes to do the same in their communities. Such efforts require external funding in a fee-for-service payment model. ( $\mathrm{J}$ Am Board Fam Med 2021;34:S170-S178.)

Keywords: COVID-19, Fee-for-Service Plans, Hotlines, Pandemics, Primary Health Care, Oregon, Telemedicine

In March 2020, the large number of people who had symptoms and questions about COVID-19 and heightened psychological ${ }^{1}$ and physical distress ${ }^{2}$ suggested a need for increased access to primary care. At the same time, the United States had a shortage of primary care. Pandemic care requires sustained, rapid change. ${ }^{3,4}$ Initially, changes were needed to avoid in-person health care encounters for virtual care-sensitive conditions. Subsequently, primary care needed plans to continue telehealth and safe strategies to bring people back to the practice. Academic health systems may be in a position

This article was externally peer reviewed.

Submitted 29 April 2020; revised 17 June 2020; accepted 18 June 2020.

From the Oregon Health \& Science University, Portland, OR (AC, HA, NH, KS, EB, EH, CM, FB, KP, DJC, JD); unaffiliated (CS, BS).

Funding: This work was supported by the philanthropy of the Andrew and Corey Morris-Singer Foundation.

Conflicts of interest: None.

Corresponding author: Anthony Cheng, MD, Oregon Health \& Science University, Portland, OR 97239 (E-mail: chengan@ohsu.edu). to augment less resource-rich primary care teams with regional telehealth services and facilitate information exchange for practice transformation. ${ }^{5}$

At the Oregon Health \& Science University (OHSU), primary care nurses discontinued routine chronic disease management functions and answered thousands of COVID-related calls in the first weeks of the pandemic. Simultaneously, clinic leaders were rapidly adapting practice operations and population health activities. Practices across the state faced similar challenges; many had fewer resources to implement rapid change. Many community members lacked access to primary care. We wanted to help. The vision for the COVID-19 Connected Care Center, a statewide telephone "hotline," was shared with philanthropic organizations and institutional leaders on March 20, 2020, funded on the March 23, and launched on March 30.

\section{Innovation}

Rapid policy changes provided financial support for the use of telemedicine platforms. The Centers for 
Figure 1. This workflow diagram illustrates how a nurse (RN) manages patient calls, which begin with a phone call to an Oregon Health \& Science University (OHSU) clinic or to the hotline's direct number. Patients with an OHSU Primary Care Provider (PCP) and with no PCP are managed within the system. Patients with a non-OHSU PCP are referred back to their provider after assessment.

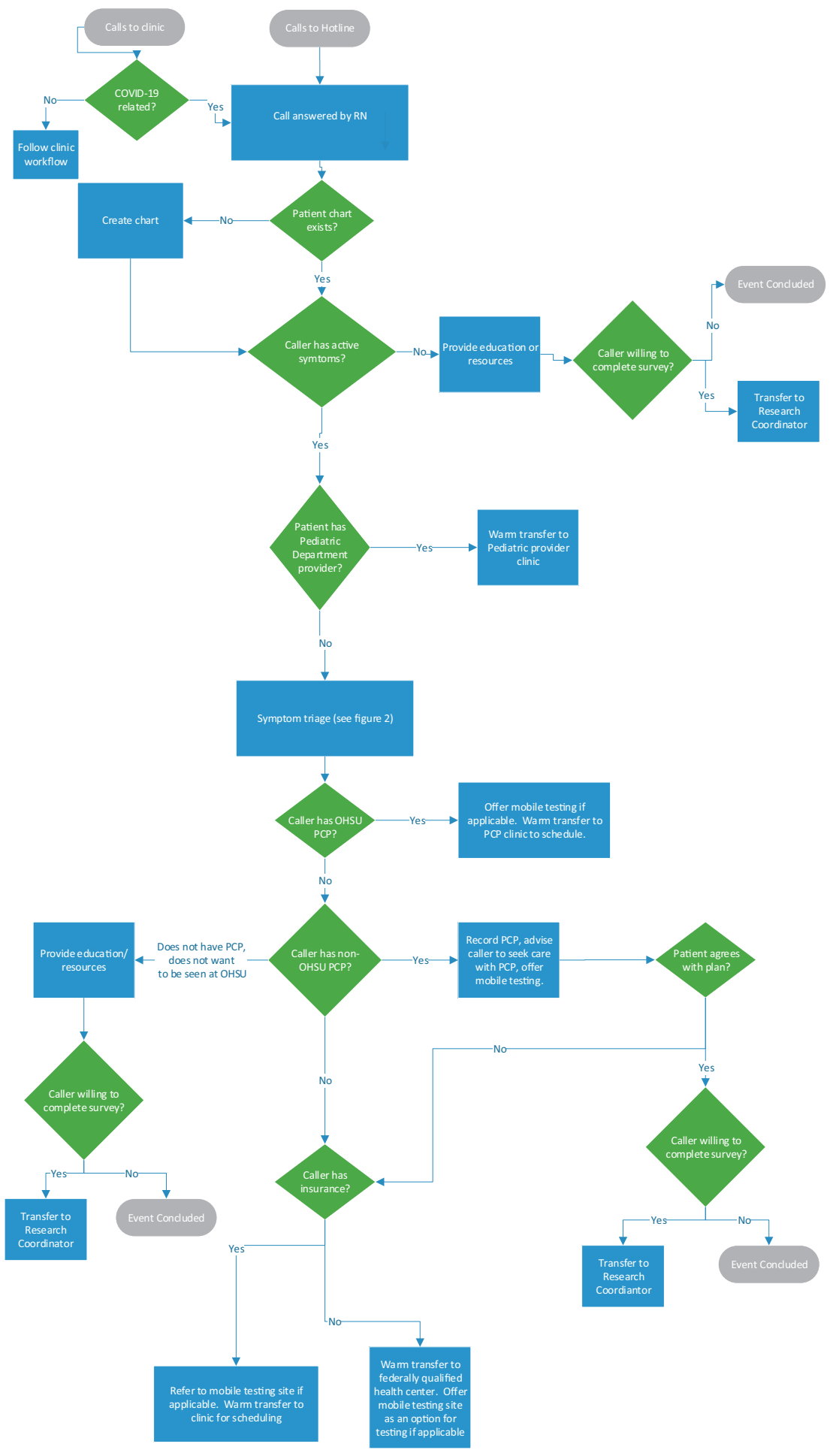


Figure 2. This diagram illustrates the management of calls after nurse triage of symptom severity as mild, moderate or severe. The primary care provider (PCP) is notified if a patient is referred to the emergency department.

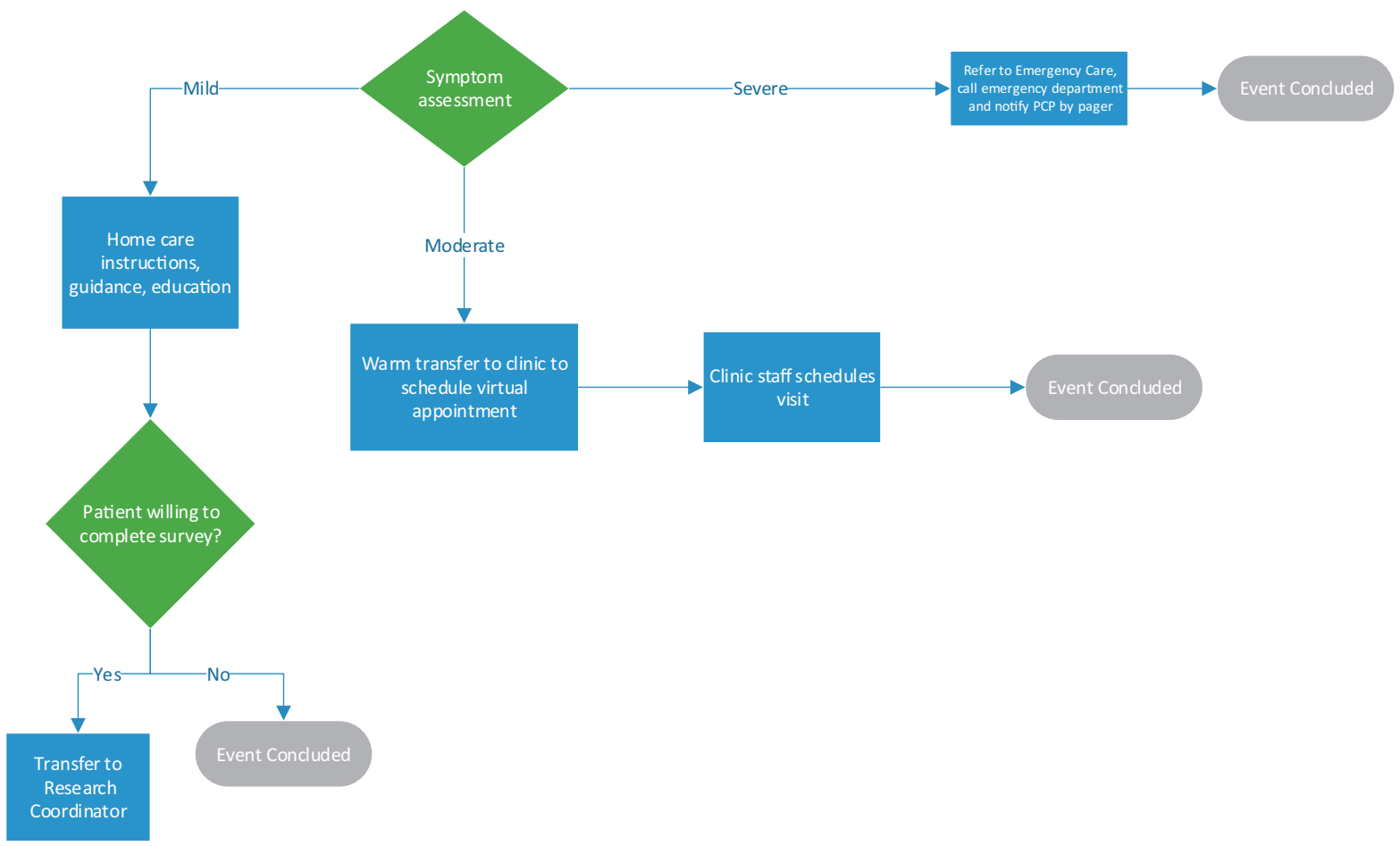

Medicare and Medicaid Services and many private payers increased payment rates for telehealth services and broadened the scope of covered services. This allowed us to link the hotline to an expanded range of digital health, primary care, and population health capabilities. The work systems and processes that we implemented are described using the SEIPS 2.0 Model, a human factors framework based on principles such as systems orientation, person-centeredness, and design-driven improvements (see Table 1). ${ }^{6,7}$ We maintained a strong evidence base, minimized handoffs in our workflows, offered virtual visits when appropriate, and arranged care to be provided by patients' primary care clinicians whenever possible. We decided that clinic staff would schedule virtual visits to allow for local variations in workflows. Home care was supported by online patient education resources and secure patient messaging portals. Almost all work of the hotline was conducted by staff working from home.

The hotline aligned efforts launched in response to the pandemic, including those to redeploy workforce and keep learners engaged. The willingness of staff and faculty to redeploy and community members' volunteer efforts were critical. We also aligned our work with community and state partners, including the Oregon Health Authority (OHA), Oregon's Poison Control Center, the Portland Fire Department, local public health departments, and 2-1-1, a nonprofit organization providing access to more than 7000 nonprofit, government, and faith-based health and social service programs.

\section{Launch Timeline}

OHSU's COVID-19 Connected Care Center was launched in 3 phases.

\section{Phase 1: OHSU Patient Hotline}

On March 30, the hotline was opened to established OHSU patients, with hours 7 days a week from 8 AM to 8 PM. Patients calling the hospital's primary care and specialty clinics had the option to be routed to the hotline, where they would speak with a triage nurse who provided medical advice about clinical symptoms, scheduled virtual care, and referred to a mobile testing site, social worker, or the emergency department. Hotline nurses managed COVID-19 test results from OHSU sites, including its mobile testing sites, called patients with positive results, and provided education. 
Figure 3. Provider or practice leaders also call the hotline. Clinical questions are answered by an on call physician. Operational questions are answered by practice leaders via email. Patient calls are managed as in Figure 1 and 2.

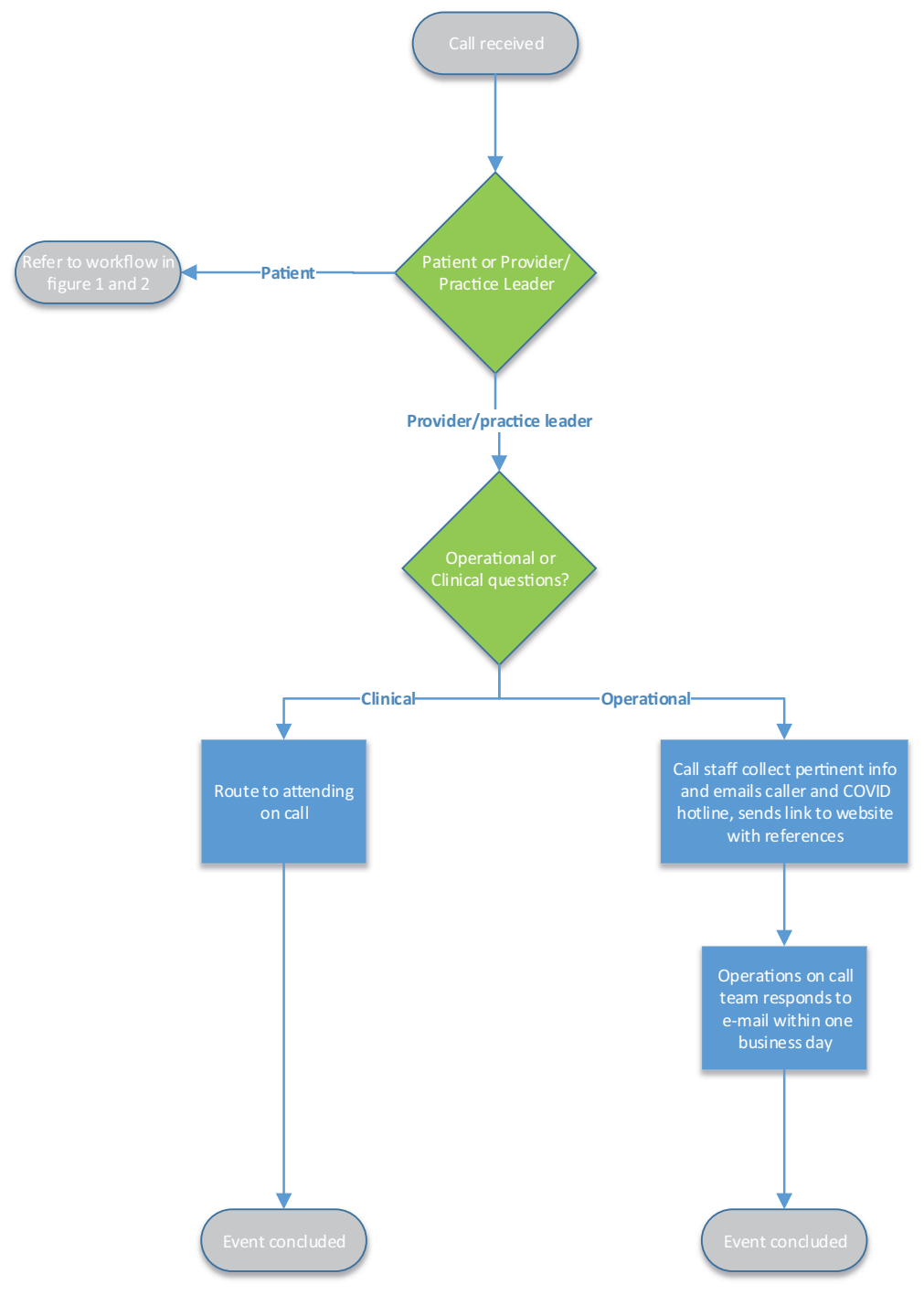

Among patients positive for COVID-19, nurses called to reassess symptoms. A small percentage of patients referred to virtual visits were then referred to our respiratory clinic for in-person evaluation. ${ }^{8}$ Patients with questions pertaining to mental health care, social, and economic services were connected with an on-call social worker or social services.

\section{Phase 2: Statewide Provider Hotline}

Beginning April 9, a central e-mail, website, and telephone consult line opened to primary care and specialty practices across Oregon. Clinicians were put in contact with an attending team, who answered symptom, testing, and operational questions.

\section{Phase 3: Statewide Patient Hotline}

Beginning April 16, the patient hotline opened to Oregonians, with the target audience being people without access to primary care. Patients requiring a visit were referred to their primary care clinician, regional practices open to new patients, or OHSU clinics. COVID-19-related care was provided free of charge. Partnerships were leveraged to reach patients; the hotline was advertised on the OHA website as part of its health care partner resource and at c19.oregon.com, the Portland Metro Emergency Response for the Coronavirus Checker online tool. Figure 1 illustrates the flow of phone calls, Figure 2 management of symptoms, and Figure 3 the handling of calls from providers and practice leaders. 
Table 1. CoVID-19 Connected Care Center Hotline Work System: Description of the CoVID-19 Connected Care Center Work System in Terms of SEIPS 2.0 Components, Including the Persons Involved, Their Tasks, the Tools and Technology They Used, and Processes Used for Adaptation

\begin{tabular}{|c|c|}
\hline Persons & Tasks \\
\hline $\begin{array}{l}\text { Implementation team: a team of } \\
\text { clinical, operations, and call } \\
\text { center experts created and } \\
\text { implemented the program. } \\
\text { Workflow development } \\
\text { sessions were led by internal } \\
\text { performance improvement } \\
\text { consultants. }\end{array}$ & $\begin{array}{l}\text { - Define and implement } \\
\text { workflows. } \\
\text { - Produce resources } \\
\text { needed to support } \\
\text { standard work. } \\
\text { - Align work with } \\
\text { institutional and } \\
\text { statewide stakeholders. }\end{array}$ \\
\hline
\end{tabular}

COVID Inquiry Group: medical students were not able to participate in clinical rotations and were offered this COVID elective course. Residents on suspended clinical rotations also participated. Clinical and research faculty lead the group along with a project coordinator and administrative staff. Librarians and specialists provided consultative backing.

Triage nurses: the team is composed of nurses from across OHSU who were reassigned from other duties.
- Continuous research to create and organize a library of materials that are accessible to all hotline teams as well as primary care and ambulatory specialty practices at OHSU and across the state.

- Update resource daily.

- Assess severity of illness and arrange appropriate disposition for patients. Disposition options include mobile testing, home care, virtual visits, referral to primary care respiratory clinic, and referral to an emergency department.

- Answer phone calls and electronic messages directed to the hotline from OHSU clinics.

- Perform symptom assessments for patients with a positive COVID19 test.
Intensive workflow development sessions were conducted in person. The remainder of meetings were conducted remotely.

Tools and Technology Adaptation

- Institutional leaders were identified and convened into a daily steering committee to provide oversight of the implementation team and to rapidly identify and address barriers. This allowed us to navigate and influence the internal and external environment. For example, the steering committee helped us quickly develop health information technology tools within OHSU and identify areas for alignment with public health agencies.

- Daily huddles occurred for continuous process improvement.

- Multiple tools were used to support the needs for large group, small group, and individual work with synchronous and asynchronous communication in both text-based and audiovisual formats.

- More information can be found about the inquiry group in a separate paper. ${ }^{10}$

- A centralized phone number and e-mail address were created for the hotline. Phone trees were reprogrammed to provide access points to the hotline from ambulatory clinics. The phone tree was integrated into other existing phone trees across the university as appropriate.

- Nurses participated in an initial 2-hour virtual training.

- Nurses were given telephones, laptops, and headsets to allow them to work from home.

- Goals of care scripting are provided for rapid assessment of patients before referral to the emergency department. secure instant messaging collaborative culture with
- Dedicated electronic health record training teams and resources were made available in real time via conferencing software to support the nurses in the first 3 weeks after hotline implementation.

- Daily huddles occur for continuous process improvement.

- Conferencing software and allow constant communication among the nurses to create a rapid dissemination of knowledge and peer-to-peer learning.

- Quality assurance activities are ongoing, including review of live and recorded calls and operational data about the hotline (eg, hold times, abandonment rates), and patient experience surveys are collected. A more formal evaluation is also being developed (see Table 2 for more details). 
Table 1. Continued

\begin{tabular}{|c|c|c|c|}
\hline Persons & Tasks & Tools and Technology & Adaptation \\
\hline $\begin{array}{l}\text { Hotline attendings: OHSU } \\
\text { physicians and advanced } \\
\text { practice providers agreed to } \\
\text { be on-call and performed this } \\
\text { work in addition to their } \\
\text { normal duties. }\end{array}$ & $\begin{array}{l}\text { - Provide consultation to } \\
\text { triage nurses and support } \\
\text { in navigating difficult } \\
\text { clinical scenarios. } \\
\text { - Answer clinical questions } \\
\text { from providers across the } \\
\text { state. }\end{array}$ & $\begin{array}{l}\text { - Provider onboarding was } \\
\text { performed remotely. } \\
\text { A dynamically updated } \\
\text { frequently asked questions } \\
\text { document was created } \\
\text { through an internal peer- } \\
\text { review process to provide } \\
\text { evidence-based answers. This } \\
\text { work was done by the OHSU } \\
\text { School of Medicine COVID- } \\
\text { 19 Inquiry Group. } \\
\text { This work was supported by } \\
\text { the COVID-19 Connected } \\
\text { Care Center Reference Guide } \\
\text { which collates workflows and } \\
\text { protocols from across the } \\
\text { university. This resource is } \\
\text { dynamically updated. }\end{array}$ & $\begin{array}{l}\text { Development of expertise was } \\
\text { encouraged by participation in } \\
\text { institutional and statewide } \\
\text { educational forums that } \\
\text { occurred weekly. }\end{array}$ \\
\hline $\begin{array}{l}\text { Ambulatory COVID-19 } \\
\text { Operations Team: clinical } \\
\text { managers and other medical } \\
\text { operations experts } \\
\text { representing medical delivery, } \\
\text { front desk staff, and clinical } \\
\text { support operations. }\end{array}$ & $\begin{array}{l}\text { - Create and update a } \\
\text { guide with information } \\
\text { about running an } \\
\text { ambulatory clinic under } \\
\text { the constraints of social } \\
\text { distancing (eg, workflows } \\
\text { to postpone visits, } \\
\text { transition to virtual } \\
\text { visits, provide education } \\
\text { to patients and } \\
\text { communities). } \\
\text { - Provide advice to } \\
\text { statewide practice leaders } \\
\text { navigating areas of } \\
\text { uncertainty. } \\
\text { Serve as a repository of } \\
\text { best practices from } \\
\text { outside institutions. }\end{array}$ & $\begin{array}{l}\text { In addition to the hotline phone } \\
\text { number, a dedicated e-mail } \\
\text { address was created for } \\
\text { primary care practices across } \\
\text { the state to send questions to } \\
\text { this group. }\end{array}$ & $\begin{array}{l}\text { Continuous review and updates } \\
\text { to COVID-19 Connected } \\
\text { Care Center Reference Guide. }\end{array}$ \\
\hline
\end{tabular}

OHSU, Oregon Health \& Science University.

In all phases, call teams were supported by an attending, and nurses and attendings referred to a shared reference, the COVID-19 Connected Care Center Reference Guide, which was developed in real time by the School of Medicine COVID-19 Inquiry Group, an "intellectual engine" providing evidence-based recommendations using rapidly evolving data. ${ }^{9}$ This group consisted of volunteer clinical and research faculty, medical students, and residents. Medical students' participation qualified as an elective. The group answered questions identified from hotline calls and clinicians participating in statewide Project ECHOs (Extension of Community Health Care Outcomes $)^{10}$ on COVID-19. ECHOs had more than 1000 registrants, $25 \%$ of whom served rural or frontier communities. The inquiry group validated information through an internal peerreview process before dissemination.

\section{Preliminary Results and Evaluation Plan}

Between March 30 and June 1, 2020 the OHSU COVID-19 Connected Care Center took more than 5825 calls from patients and providers in 33 of Oregon's 36 counties. There were an average of 141 triage calls and 68 results calls each weekday. The team also handled 9594 results and 916 patient email messages. An average triage call was 6 minutes; an average results call was 30 minutes. Of the patient callers, approximately $36 \%$ received education; $49 \%$ had mild, $19 \%$ had moderate, and $1 \%$ had severe symptoms. In the first 2488 calls, only 7 resulted in an emergency care referral. Twenty-four full-time nurses staffed the hotline. In subsequent months, call volumes increased; total calls exceeded 60,000 by the end of the year.

To evaluate the Connected Care Center, we are using the RE-AIM Framework (Reach, Effectiveness, 
Table 2. CovID-19 Connected Care Center Evaluation Research Questions, Measures, and Data Collection Approach

RE-AIM Element and Research Question (RQ)

Measures/Data Collection Approach

Reach — the absolute number, proportion, and representativeness of individuals who are willing to participate in a given initiative RQ: To what extent does the hotline reach the intended target population?

Effectiveness-the impact of an intervention on important outcomes, including potential negative effects.

RQ: Does the telephone hotline impact key outcomes?

Primary outcomes:Patient and provider experience

Were questions answered?Did patients feel reassured?

Would patients/providers recommend this service?

How much do patients value the service?

Clinical outcomes and care use

- Was testing offered when indicated.

- Follow-up rate.

- In-person visit/video visit-follow-up rate.

- Appropriate alignment of care level with severity-accuracy based on peer review.

- Infection prevention-adherence to quarantine, isolation, return to work recommendations.

- Emergency department-admission rates, total care cost.

Adoption-willingness and experience of staff implementing the hotline.

Implementation—at the setting level, implementation refers to the intervention agents' fidelity to the various elements of an intervention's protocol. This includes consistency of delivery as intended and the time and cost of the intervention.

RQ: How was the hotline implemented? What were the key elements of the "intervention" and what processes were put in place to ensure fidelity of the hotline?

Maintenance-the extent to which a program or policy becomes institutionalized or part of the routine organizational practices and policies.RQ: Among key stakeholders, how would the hotline (if it is needed) be maintained, and what is needed to maintain it?
Numerators: number of target population for telephone hotline that called and spoke to someone; includes describing the following: trend in use over time, description of user (OHSU patient or not, sex, age), user patterns (time of the day), user location.

Denominator: number of of OHSU patients and number of Oregonians without a primary care provider.

Source: data from phone system, demographics for OHSU patients, statewide estimate of patients without a primary care provider.

Survey data: post-telephone surveys will be conducted with patients. Post-e-mail and post-telephone surveys will be conducted with providers.

Qualitative interviews: these can be used to explore patient experiences more deeply and to try to understand variations in outcomes. Specifically, we will examine other information seeking and support seeking, access to services, alignment of the center with patients' and providers' needs, the community value of the call center, and access from both patients' and providers' perspectives.

Source: electronic health records, Medicaid claims, statewide data.

Source: semistructured interviews with staff to understand enjoyment of role, perceived readiness for role after training, responsibilities consistent with top of licensure, enjoyment of other nonclinical peers, perceived value of the hotline, confidence in the quality, and impact on well-being.

Source: qualitative data collection; interviews with key stakeholders: this will include understanding the staffing and functionality of the hotline, the staff full-time equivalent (FTE) and change in staff FTE over time, the type of staff involved, barrier/facilitators to implementation (eg, opportunities, technical issues, drop calls, hang up, hold times, and how these were resolved), partnership with the state and others that made this work possible.

Source: semistructured interviews with key stakeholders, after the active implementation phase of the pilot has ended, to assess factors related to maintenance.

RE-AIM, Reach, Effectiveness, Adoption, Implementation, and Maintenance; OHSU, Oregon Health \& Science University.

Adoption, Implementation, and Maintenance) to answer the following questions: (1) Did the Connected Care Center reach the intended target populations? (2) Was the Connected Care Center effective? (3) What can we learn about what worked and what did not to refine this effort? (4) What aspects of the Connected Care Center can and should be maintained, and how? To answer these questions, we are collecting quantitative and qualitative data as shown in Table 2. Data collection is ongoing.

Preliminary data show that patients called the hotline seeking personalized information to translate publicly available information to their own situation. As a result of their call, patients reported feeling their stress and anxiety about COVID-19 reduced, receiving information and access to care and testing and education that included help developing plans to social distance to protect coworkers and family members from transmission. When surveyed $(\mathrm{n}=50), 86 \%$ of patients reported that their questions were answered during the call, and $90 \%$ said they would recommend this service to a friend or family member. 


\section{Discussion}

\section{Evaluation Plan}

A nurse triage hotline to address patients concerns about the COVID-19 pandemic can relieve a substantial burden from primary care practices ${ }^{11}$ and contribute to efforts to prevent mildly sick but contagious people from seeking in-person care. The majority of patients that called our hotline required education only, and nurses talked to patients with mild symptoms, which were appropriately handled by a nurse telephonically.

In addition to patients, practice leaders from almost every Oregon county used the hotline to inform how they managed COVID-19. We shared resources, including almost 450 "frequently asked questions" and a reference guide, and have fielded 320 calls and 28 e-mails from primary care clinicians and practice leaders (through June 6, 2020). OHSU was a conduit for rapidly assimilating evidence and providing direct support to primary care practices across the state, helping them optimize patient care during this pandemic. ${ }^{12-19}$ Involving learners allows them to contribute to the pandemic response, safely. This centralized team of experts has also facilitated research and community based testing. Thus, the hotline served several of the functions envisioned for a primary care extension program, an unfunded mandate of the 2011 Patient Protection and Affordable Care Act. Where the federal government left a funding gap, the Andrew and Corey Morris-Singer Foundation stepped in and made a generous donation. Our experience demonstrates how a state-based primary care extension model, if funded, can support pandemic preparedness.

Our next step is to fully evaluate this effort, including identifying factors that led to implementation success (eg, alignment of institutional leadership, ability to adapt, visibility of the work across multiple levels of the institution) and determine which elements of this approach were most effective and why. Of particular interest is the impact of the hotline on primary care teams in community practice, particularly in rural and underserved areas, and to understand the impact on and the best strategies for reaching marginalized populations who suffer disproportionately from COVID-19. This includes taking steps to expand our partnerships to include community-based organizations and community leaders with diverse perspectives. ${ }^{20}$

\section{Conclusion}

With adequate resources and funding, it is possible to rapidly implement a multiphase coordinated approach to aid primary care teams on a statewide level to respond to a pandemic. The COVID-19 Connected Care Center addressed patients', community members', and providers' COVID-related concerns. The hotline is an example of how an academic health center can support public health and statewide practice adaptation.

A generous donation from the Andrew and Corey MorrisSinger Foundation supported the establishment of an OHSU phone hotline and telemedicine service to enable many more Oregonians to access the COVID-19 primary care resources and information. We wish to thank the team of leaders, managers, and operations and logistics experts who worked 24/7 behind the scenes to make all of this happen. Those not already named include our executive sponsors John Hunter, MD, Bridget Barnes, MBA, MSEM, and Renee Edwards, MD. We also wish to thank steering committee cochair Kevin O'Boyle, MHA and lead for operations Debbie Lamberger, MPA. This work would not have been possible without the help of project managers Shilpa Kasukurthi, Ali Bruni, and Jessica Hoyt. We appreciate the guidance from steering committee members Johanna Warren, MD, Sarah Present, MD, MPH, Cat Livingston MD, MPH, Darren Malinowski, MD, Steve Kassakian, MD, Banning Hendriks, MBA, Betsy Boyd-Flynn, and Kelsey Huwaldt, MBA.

To see this article online, please go to: http://jabfm.org/content/ 34/Supplement/S170.full.

\section{References}

1. Tan R. In an era of quarantine, crisis hotlines face growing — and urgent-demand. Washington Post. March 23, 2020. Available from: https://www. washingtonpost.com/local/crisis-hotline-quarantinecoronavirus-mental-health/2020/03/23/632e2d7c6abe-11ea-9923-57073adce27c_story.html. Accessed April 15, 2020.

2. Keeter S. People financially affected by COVID-19 outbreak are experiencing more psychological distress than others. Pew Research Center Policy Brief. March 3, 2020. Available from: https://www.pewresearch.org/ fact-tank/2020/03/30/people-financially-affected-bycovid-19-outbreak-are-experiencing-more-psychologicaldistress-than-others/. Accessed April 15, 2020.

3. Mehotra A, Ray K, Brockmeyer DM, Barnett ML, Bender JA. Rapidly converting to "virtual practices": outpatient care in the era of Covid-19. N Engl J Med Catalyst April 1, 2020. doi: 10.1056/CAT.20.0091.

4. Krist A, DeVoe J, Cheng A, Ehrlich T. The five phases of pandemic care for primary care. Ann Fam Med, COVID-19 Collection. 2020. Available from: http://hdl.handle.net/2027.42/154687.

5. DeVoe JE, Cheng A, Krist A. Regional strategies for academic health centers to support primary care 
during the COVID-19 pandemic. JAMA Health Forum April 8, 2020;1:e200423. doi:http://dx.doi. org/10.1001/jamahealthforum.2020.0423.

6. Holden RJ, Carayon P, Gurses AP, et al. SEIPS 2.0: a human factors framework for studying and improving the work of healthcare professionals and patients. Ergonomics October 2013;56:1669-2020.

7. Reason J. Human error: models and management. BMJ 2000;320:768-70.

8. McDougall C, Joslin T, Yamashita D, Barnett A, Rugge B, DeVoe JD. We built a primary care respiratory clinic. Here's how it works. April 22, 2020. Available from: https://medium.com/case-notes-fromthe-covid-19-front-lines/we-built-a-primary-carerespiratory-clinic-heres-how-it-works-3e2abb02886f. Accessed April 27, 2020.

9. Robinson S, Angier H, Pierce K, et al. Responding to community questions during a pandemic: development of a COVID-19 inquiry group. Ann Fam Med, COVID-19 Collection. 2020.

10. Steeves-Reece AL, Elder NC, Graham TA, et al. Rapid deployment of a statewide COVID-19 ECHO program for frontline clinicians: early results and lessons learned. Journal of Rural Health 2020;May. doi: 10.1111/jrh.12462.

11. Patient-Centered Primary Care Collaborative. "Clinician survey." Quick Covid-19 survey. March 13, 2020. Available from: https://static1.squarespace.com/static/ $5 \mathrm{~d} 7 \mathrm{ff} 8184 \mathrm{cf0e} 01 \mathrm{e} 4566 \mathrm{cb} 02 / \mathrm{t} / 5 \mathrm{e} 861 \mathrm{fc} 4 \mathrm{f} 218 \mathrm{f} 93 \mathrm{aefa} 2$ eb7a/1585848260602/C19+Series + 1+PC+Survey+ COVID19+Mar+13-16+Summary.pdf. Accessed June 6, 2020.
12. Ono SS, Crabtree BF, Hemler JR, et al. Taking innovation to scale in primary care practices: the functions of healthcare extensions. Health Aff (Millwood) 2018;37:222-30.

13. Kaufman A, Dickinson WP, Fagnan LJ, Duffy FD, Parchman ML, Rhyne RL. The role of health extension in practice transformation and community health improvement: lessons from 5 case studies. Ann Fam Med 2019;17:S67-S72.

14. Grumbach K, Mold JW. A health care cooperative extension service: transforming primary care and community health. JAMA 2009;301:2589-91.

15. Kaufman A, Powell W, Alfero C, et al. Health extension in New Mexico: an academic health center and the social determinants of disease. Ann Fam Med 2010;8:73-81.

16. Kaufman A, Boren J, Koukel S, Ronquillo F, Davies C, Nkouaga C. Agriculture and health sectors collaborate in addressing population health. Ann Fam Med 2017;15:475-80.

17. Mold JW. A cooperative extension service for primary care in Oklahoma. J Okla State Med Assoc 2011;104:414-8.

18. Fagnan LJ. Moving upstream-health extension and primary care. J Am Board Fam Med 2017;30:10-2.

19. Phillips RL, Cohen DJ, Kaufman A, Dickinson WP, Cykert S. Facilitating practice transformation in frontline health care. Ann Fam Med 2019;17:S2 S5. doi:10.1370/afm.2439.

20. Kantameneni N. The impact of the COVID-19 pandemic on marginalized populations in the United States: a research agenda.J Vocat Behav 2020;119:103439. 